\title{
Simplified Preoperative Assessment for Appendix Tumor Score
}

National Cancer Institute

\section{Source}

National Cancer Institute. Simplified Preoperative Assessment for Appendix Tumor

Score. NCI Thesaurus. Code C141410.

A set of imaging criteria, based on preoperative CT scans, used to predict the likelihood for complete cytoreduction in patients with low-grade mucinous adenocarcinoma (LGMA) of the appendix. A score is determined by the presence of scalloping at 4 anatomic sites and the absence or presence of mesenteric foreshortening of the small bowel. A score under 3 is a significant predictor for complete cytoreduction. 